\title{
Rotational symmetry breaking in baby Skyrme models
}

\author{
I Hen and M Karliner \\ School of Physics and Astronomy, Raymond and Beverly Sackler Faculty of Exact \\ Sciences, Tel-Aviv University, Tel-Aviv 69978, Israel.
}

E-mail: itayhe@post.tau.ac.il, marek@neutron.tau.ac.il

\begin{abstract}
We consider multisolitons with charges $1 \leq B \leq 5$ in the baby Skyrme model for the one-parametric family of potentials $U=\mu^{2}\left(1-\phi_{3}\right)^{s}$ with $0<s \leq 4$. This class of potentials is a generalization of the 'old' $(s=1)$ and 'holomorphic' $(s=4)$ baby Skyrme models. We find that for charge one, stable solutions exist for every value of $s$ and they are rotationally-symmetric. For higher charges, stable solutions exist only below $s \approx 2$. In the charge-two sector the stable solutions are always rotationallysymmetric and ring-like. For charge three and above, rotational symmetry is exhibited only in the small $s$ region; above a certain critical value of $s$, this symmetry is broken and a strong repulsion between the constituent one-Skyrmions becomes apparent. We also compute the spatial energy distributions of these solutions.
\end{abstract}

PACS numbers: 0350, 1110

Submitted to: Nonlinearity 


\section{Introduction}

The Skyrme model [1, 2] is an SU(2)-valued non-linear theory for pions in $(3+1)$ dimensions with topological soliton solutions called Skyrmions. Apart from a kinetic term, the Lagrangian of the model contains a 'Skyrme' term which is of the fourth order in derivatives, and is used to introduce scale to the model [3]. The existence of stable solutions in the Skyrme model is a consequence of the nontrivial topology of the mapping $\mathcal{M}$ of the physical space into the field space at a given time, $\mathcal{M}: S^{3} \rightarrow S U(2) \cong S^{3}$, where the physical space $\mathbb{R}^{3}$ is compactified to $S^{3}$ by requiring the spatial infinity to be equivalent in each direction. The topology which stems from this one-point compactification allows the classification of maps into equivalence classes, each of which has a unique conserved quantity called the topological charge.

The Skyrme model has an analogue in $(2+1)$ dimensions known as the baby Skyrme model, also admitting stable field configurations of a solitonic nature [5]. Due to its lower dimension, it serves as a simplification of the original model, but nonetheless it has a physical significance on its own, having several potential applications in condensedmatter physics [6]. The target manifold in the baby model is described by a threedimensional vector $\phi$ with the constraint $\phi \cdot \phi=1$. In analogy with the $(3+1) \mathrm{D}$ case, the domain of this model $\mathbb{R}^{2}$ is compactified to $S^{2}$ yielding the topology required for the classification of its field configurations into classes with conserved topological charges. The Lagrangian density of the baby Skyrme model is given by:

$\mathcal{L}=\frac{1}{2} \partial_{\mu} \boldsymbol{\phi} \cdot \partial^{\mu} \boldsymbol{\phi}-\frac{\kappa^{2}}{2}\left[\left(\partial_{\mu} \boldsymbol{\phi} \cdot \partial^{\mu} \boldsymbol{\phi}\right)^{2}-\left(\partial_{\mu} \boldsymbol{\phi} \cdot \partial_{\nu} \boldsymbol{\phi}\right) \cdot\left(\partial^{\mu} \boldsymbol{\phi} \cdot \partial^{\nu} \boldsymbol{\phi}\right)\right]-U\left(\phi_{3}\right)$

and consists analogously of a kinetic, the Skyrme and a potential term.

While in $(3+1)$ dimensions the latter term is optional [4], its presence in the $(2+1) \mathrm{D}$ model is necessary for the stability of the solutions. Aside from the requirement that the potential vanishes at infinity for a given vacuum field value (normally taken to be $\left.\boldsymbol{\phi}^{(0)}=(0,0,1)\right)$, its exact form is arbitrary and gives rise to a rich family of possible baby-Skyrme models. Three specific potentials have been studied in detail in the literature. The simplest is the holomorphic model with $U\left(\phi_{3}\right)=\mu^{2}\left(1-\phi_{3}\right)^{4}$ [7, 8, 9]. It is known to have a stable solution only in the charge-one sector. The model with the potential $U\left(\phi_{3}\right)=\mu^{2}\left(1-\phi_{3}\right)$ (commonly referred to as the 'old' model) has also been extensively studied. This potential gives rise to very structured non-rotationallysymmetric multi-Skyrmions [5, 10]. Another model with $U\left(\phi_{3}\right)=\mu^{2}\left(1-\phi_{3}^{2}\right)$ produces ring-like multi-Skyrmions [11]. Other double-vacuum potentials which give rise to other types of solutions have been studied in [12].

The brief review given above indicates that the form of the potential term has a decisive effect on the properties of the minimal energy configurations of the model. To gain better insight into this matter, in this paper we study the multisolitons of the baby Skyrme model for the one-parametric family of potentials $U=\mu^{2}\left(1-\phi_{3}\right)^{s}$ with $0<s \leq 4$ which generalizes previously studied cases. Setting $s=1$ yields the "old" model, whereas $s=4$ corresponds to the holomorphic model. Although at this time 
the physical motivation for these generalizations is not yet fully clear, we show that it nonetheless yields interesting results. The value of the parameter $s$ has dramatic effects on the static solutions of the model, both quantitatively and qualitatively, in the sense that it can be viewed as a 'control' parameter responsible for the repulsion or attraction between Skyrmions.

\section{Method}

In the present work, we find the static multisolitons of the baby Skyrme model whose Lagrangian density is given by:

$\mathcal{L}=\frac{1}{2} \partial_{\mu} \boldsymbol{\phi} \cdot \partial^{\mu} \boldsymbol{\phi}-\frac{\kappa^{2}}{2}\left(\left(\partial_{\mu} \boldsymbol{\phi} \cdot \partial^{\mu} \boldsymbol{\phi}\right)^{2}-\left(\partial_{\mu} \boldsymbol{\phi} \cdot \partial_{\nu} \boldsymbol{\phi}\right) \cdot\left(\partial^{\mu} \boldsymbol{\phi} \cdot \partial^{\nu} \boldsymbol{\phi}\right)\right)-\mu^{2}\left(1-\phi_{3}\right)^{s}$.

The model contains three free parameters, namely $\kappa, \mu$ and $s$. Since either $\kappa$ or $\mu$ may be scaled away, the parameter space of this model is in fact only two dimensional. Our main goal in this paper is to study the effects of these parameters on the static solutions of the model within each topological sector.

The multi-Skyrmions of our model are those field configurations which minimize the static energy functional within each topological sector. In polar coordinates the energy functional is given by

$E=\int r \mathrm{~d} r \mathrm{~d} \theta\left(\frac{1}{2}\left(\partial_{r} \boldsymbol{\phi} \cdot \partial_{r} \boldsymbol{\phi}+\frac{1}{r^{2}} \partial_{\theta} \boldsymbol{\phi} \cdot \partial_{\theta} \boldsymbol{\phi}\right)+\frac{\kappa^{2}}{2} \frac{\left(\partial_{r} \boldsymbol{\phi} \times \partial_{\theta} \boldsymbol{\phi}\right)^{2}}{r^{2}}+\mu^{2}\left(1-\phi_{3}\right)^{s}\right)$.

The Euler-Lagrange equations derived from the energy functional (3) are nonlinear $P D E$ 's, so in most cases one must resort to numerical techniques in order to solve them. In our approach, the minimal energy configuration of a baby Skyrmion of charge $\mathrm{B}$ and a given set of values $\mu, \kappa, s$ is found by a full-field relaxation method, described in more detail below.

One of our objectives is the study of spontaneous breaking of rotational symmetry by the static solutions. To avoid a possible bias from the use of a rectangular grid in cases in which the rotational symmetry of the solution is broken, a polar grid has been used - 120 grid points for the radial coordinate and 120 points for the angular coordinate. For numerical convenience, the physical $\mathbb{R}^{2}$ plane is further compactified into the unit circle by transforming to a truncated radial coordinate

$$
\tilde{r}=\frac{2}{\pi} \tan ^{-1}(r / \alpha)
$$

with $\alpha=1.2$. The relaxation process begins by initializing the field triplet $\phi$ to a rotationally-symmetric configuration

$$
\phi_{\text {initial }}=(\sin f(r) \cos B \theta, \sin f(r) \sin B \theta, \cos f(r)),
$$

where $B$ is the topological charge of the baby-Skyrmion in question and the profile function $f(r)$ is set to $f(r)=\pi \exp (-r)$.

The energy of the baby-Skyrmion is then minimized by repeating the following steps: a point $\left(\tilde{r}_{m}, \theta_{n}\right)$ on the grid is chosen at random, along with one of the three 
components of the field $\phi\left(\tilde{r}_{m}, \theta_{n}\right)$. The chosen component is then shifted by a value $\delta_{\phi}$ chosen uniformly from the segment $\left[-\Delta_{\phi}, \Delta_{\phi}\right]$ where $\Delta_{\phi}=0.1$ initially. The field triplet is then normalized and the change in energy is calculated. If the energy decreases, the modification of the field is accepted and otherwise it is discarded. The procedure is repeated while the value of $\Delta_{\phi}$ is gradually decreased throughout the procedure. This is done until no further decrease in energy is observed.

One undesired feature of this minimization scheme is that it can get stuck at a local minimum. This problem can be resolved by using the "simulated annealing" algorithm [13, 14], which in fact has been successfully implemented before, in obtaining the minimal energy configurations of static two and three dimensional Skyrmions [15]. The algorithm comprises of repeated applications of a Metropolis algorithm with a gradually decreasing temperature, based on the fact that when a physical system is slowly cooled down, reaching thermal equilibrium at each temperature, it will end up in its ground state. This algorithm, however, is much more expensive in terms of computer time. We therefore employ it only on a representative sample of the parameter space, just as a check on our results, which correspond to a Metropolis algorithm of zero temperature.

As a further verification, we set up the minimization scheme using different initial configurations and grids of different sizes $(60 \times 60$ and $90 \times 90)$ for several $\kappa, \mu$ and $s$ values. This was done to make sure that the final configurations are independent

of the discretization and cooling scheme. Accuracy was also verified by checking conservation of the topological charge $B$ throughout the minimization process, yielding $\left|\frac{B_{\text {observed }}}{B}-1\right|<10^{-5}$.

\section{Results}

Using the minimization procedure presented in the previous section, we have found the static solutions of the model for $1 \leq B \leq 5$. Since the parameter space of the model is effectively two dimensional (as discussed in section 2), without loss of generality we fixed the potential strength at $\mu^{2}=0.1$ throughout, and scanned the $s-\kappa$ parameter space in the region $0<s \leq 4$ and $0.01 \leq \kappa^{2} \leq 1$.

\subsection{Charge-one Skyrmions}

In the charge-one sector, stable rotationally-symmetric solutions were found for all tested values of $s$ and $\kappa$. Figure 1 shows the obtained profile functions of the $B=1$ solution for different values of $s$ with $\kappa$ fixed at $\kappa^{2}=0.25$.

Interestingly, the Skyrmion energy as function of $s$ is not monotonic, but acquires a minimum at $s \approx 2.2$, as is shown in figure 2 . 


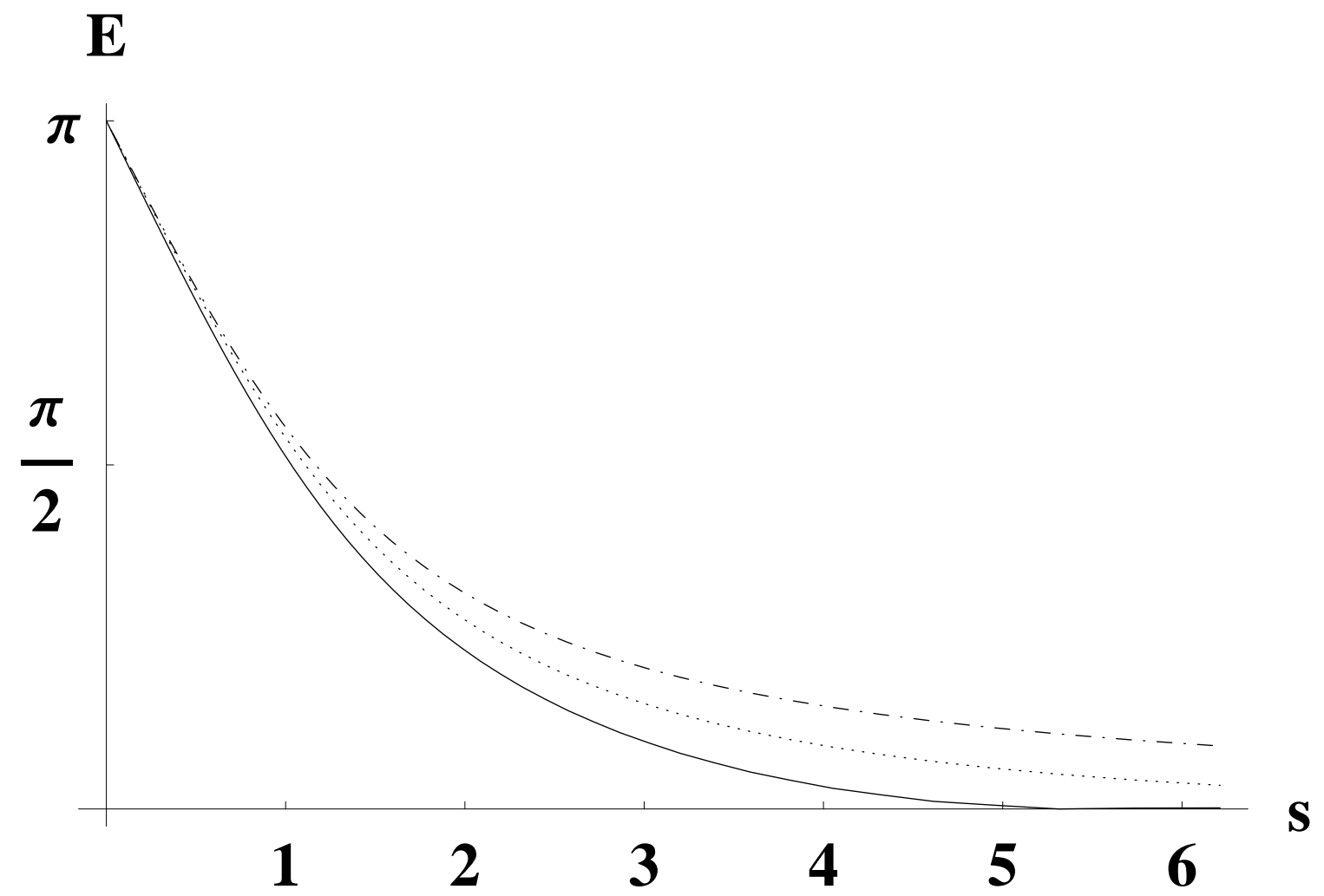

Figure 1: Profile functions of the $B=1$ Skyrmion for $s=0.5(-), s=1(\cdots \cdots)$ and $s=2(-\cdot-)$. Here $\kappa$ is fixed at $\kappa^{2}=0.25$.

\subsection{Charge-two Skyrmions}

Stable solutions were also found to exist in the $B=2$ sector, but only for $s<2$. They are rotationally-symmetric and ring-like, corresponding to two charge-one Skyrmions on top of each other. Figure 3 shows the profile functions of the stable solutions for different values of $s$ and $\kappa^{2}=0.25$.

As in the $B=1$ case, the energy of the charge-two Skyrmion as function of $s$ is non-monotonic and has a minimum in $s$ around $s=1.3$. As shown in figure 2 , at $s \approx 2$ the energy of the ring-like configuration reaches the value of twice the energy of the charge-one Skyrmion and stable configurations cease to exist. At this point, the Skyrmion breaks apart into its constituent charge-one Skyrmions, which in turn start drifting away from each other. Contour plots of the energy distribution of the $B=2$ Skyrmion are shown in figure 4 for $\kappa^{2}=1$ and for two $s$ values. While for $s=1.5$ a ring-like stable configuration exists (figure 4a), for $s=2.6$ the Skyrmion breaks apart. The latter case is shown in figure 4b which was taken while the distance between the individual Skyrmions kept growing.

These results are in accord with corresponding results from previously known studies of both the 'old' $(s=1)$ model in which ring-like configurations have been observed [5, 10], and the holomorphic model for which no stable solutions have been 


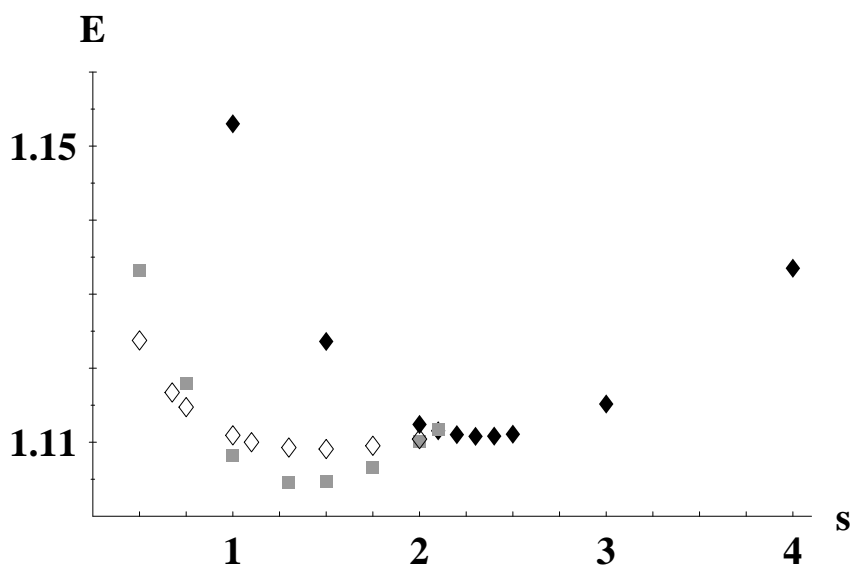

(a) $\kappa^{2}=0.05$

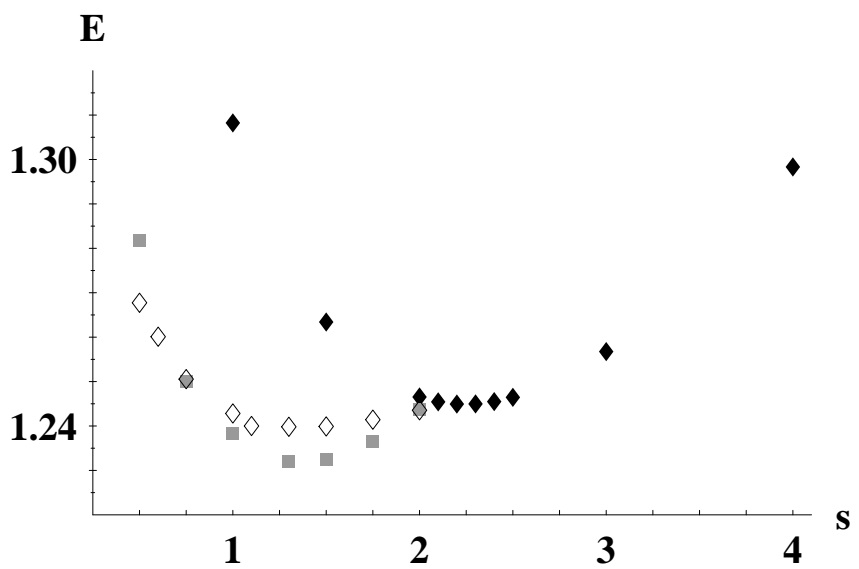

(b) $\kappa^{2}=0.25$

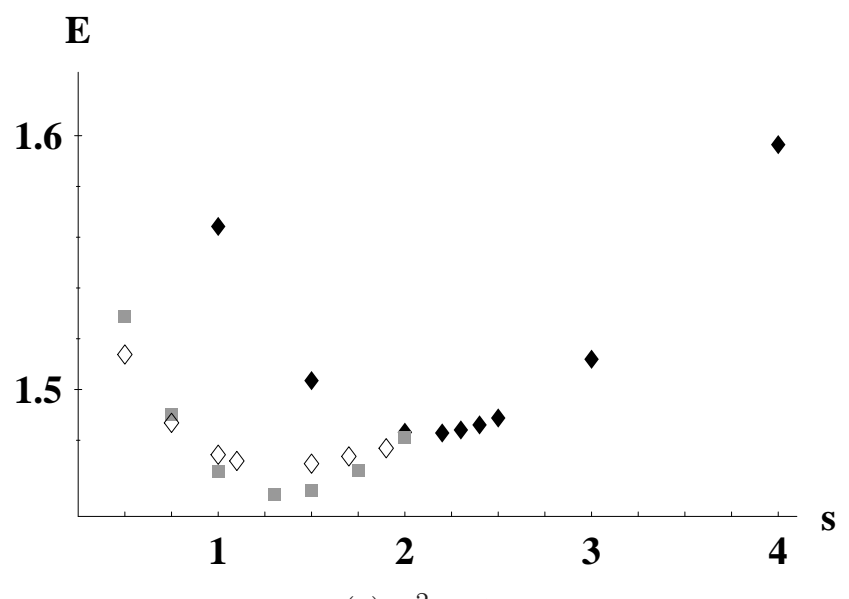

(c) $\kappa^{2}=1$

Figure 2: Total energies (divided by $4 \pi B$ ) of the charge-one $(\diamond)$ charge-two $(\square)$ and charge-three $(\diamond)$ Skyrmions as a function of the parameter $s$ for various $\kappa$ values. Each of the energy graphs attains a minimal value at some $s$. At $s \approx 2$ the energy-pertopological-charge of the charge-two and charge-three solutions reaches the charge-one energy (from below), and stable solutions are no longer observed. 
found [7, 8].

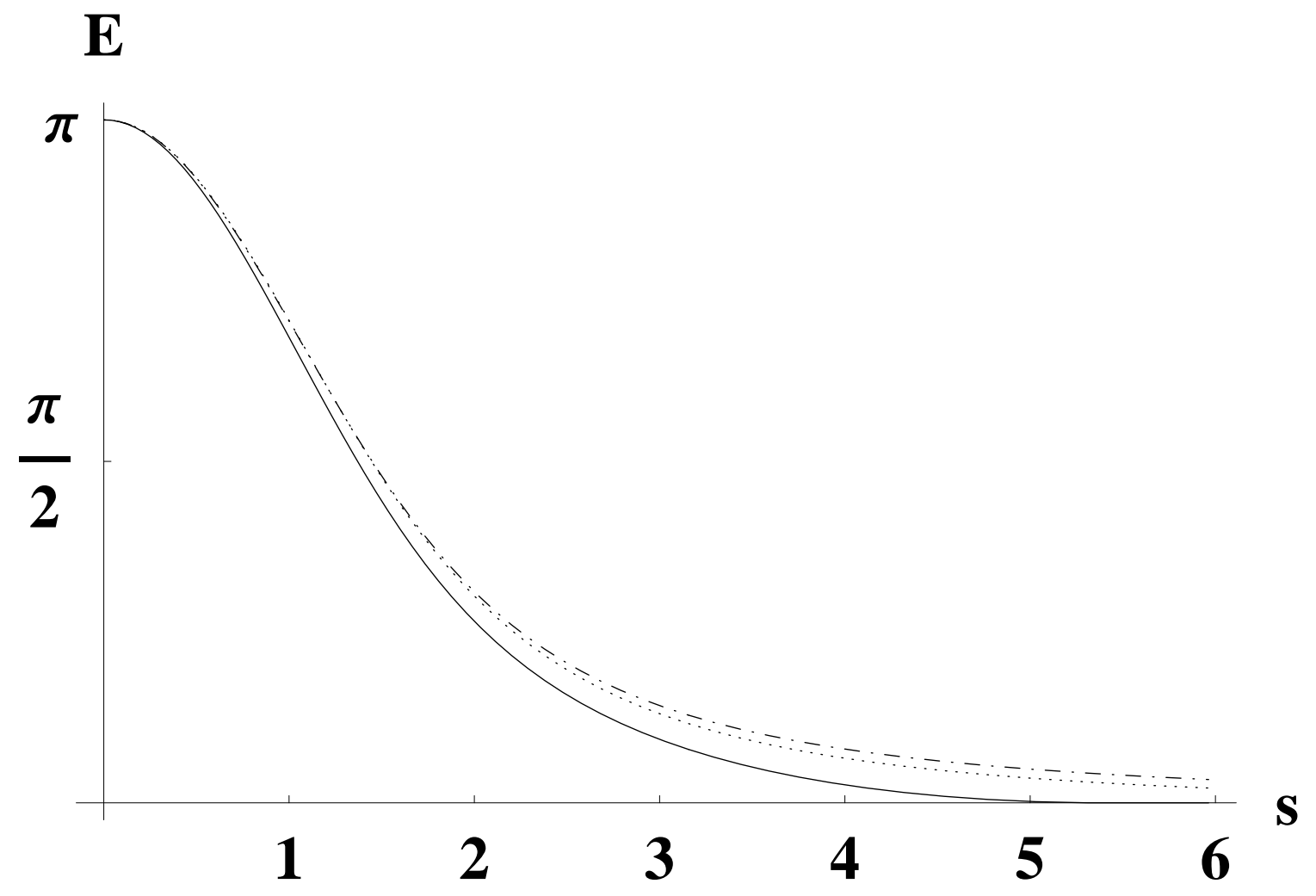

Figure 3: Profile functions of the $B=2$ Skyrmion for $s=0.5(-), s=1(\cdots \cdots)$ and $s=2(-\cdot-) . \kappa$ is fixed at $\kappa^{2}=0.25$.

We have also observed rotationally-symmetric locally stable solutions in the large $s$ regime, including the 'holomorphic' $s=4$ case. The global minimum in this regime corresponds to two infinitely separated charge-one Skyrmions. The total energy of the rotationally symmetric solutions was found to be larger than twice the energy of a charge-one Skyrmion, indicating that the split Skyrmion is an energetically more favourable configuration. We discuss this issue in more detail in the section 4 .

\subsection{Charge-three and higher-charge Skyrmions}

As with the $B=2$ Skyrmion, the existence of stable charge-three Skyrmions was also found to be $s$ dependent. For any tested value of $\kappa$ in the range $0.01 \leq \kappa^{2} \leq 1$, we have found that above $s \approx 2$, no stable charge-three solutions exist; in this region the Skyrmion breaks apart into individual Skyrmions drifting further and further away from each other.

In the $s<2$ region, where stable solutions exist, the energy distribution of the charge-three Skyrmion was found to be highly dependent on both $s$ and $\kappa$. Keeping $\kappa$ fixed and varying $s$, we have found that in the small $s$ regime, ring-like rotationallysymmetric configurations exist. Increasing the value of $s$ resulted in stable minimal 


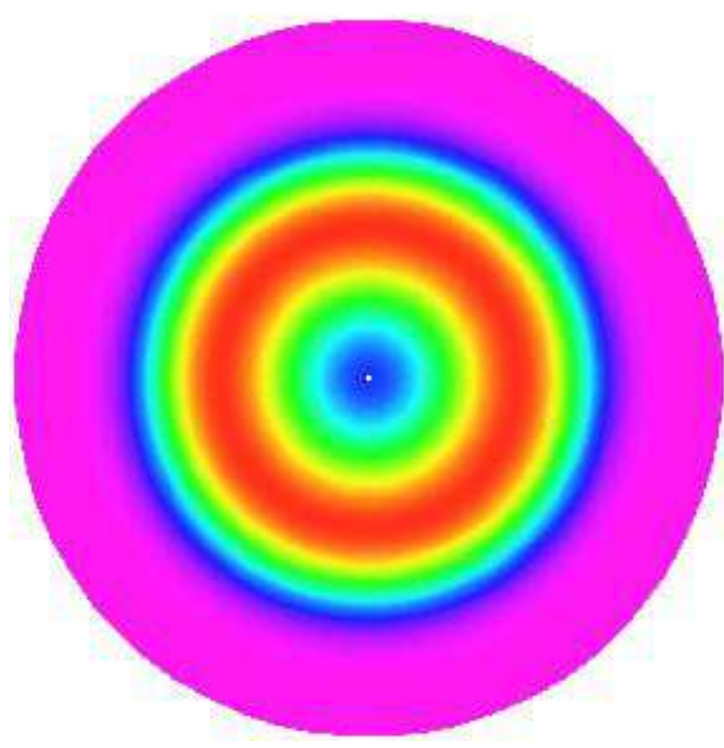

(a) $s=1.5$

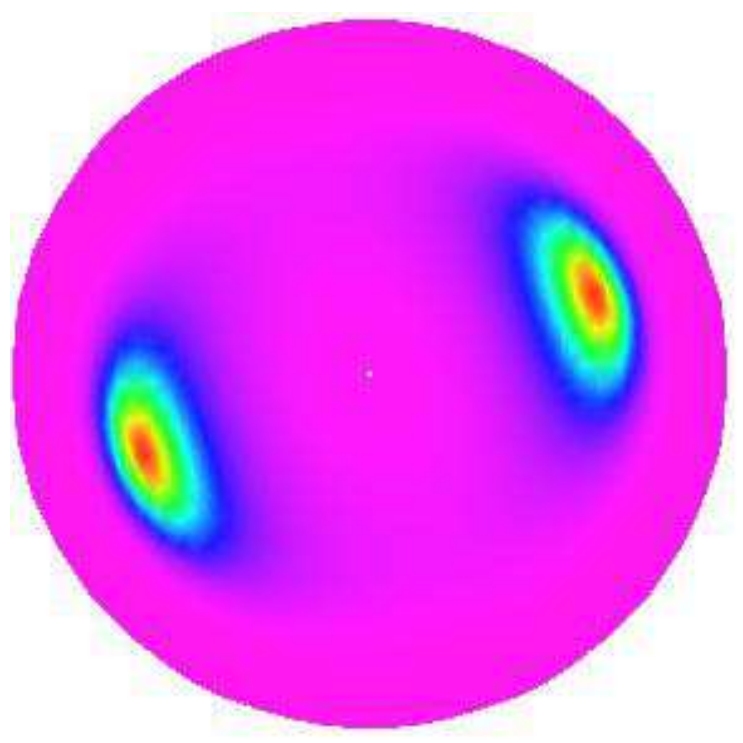

(b) $s=2.6$

Figure 4: Contour plots of the energy distributions (ranging from violet - low density to red - high density) of the $B=2$ Skyrmion for $\kappa^{2}=1$. The energy is plotted as function of the angle $\theta$ and of the scaled radial coordinate $\tilde{r}$ (eq. (4)). In the $s<2$ regime, ring-like rotationally-symmetric configurations exist, corresponding to two charge-one Skyrmions on top of each other (left), whereas for $s>2$, the charge-two Skyrmion splits into two one-charge Skyrmions drifting infinitely apart (right).

energy configurations with only $\mathbb{Z}(2)$ symmetry, corresponding to three partiallyoverlapping charge-one Skyrmions in a row, as shown in figures 5b and 5c. The energy of the charge-three Skyrmion also has a minimum in $s$, at around $s=1.5$ (As shown in figure 21). At $s \approx 2$ the energy of the Skyrmion becomes larger than three times the energy of a charge-one Skyrmion and stable configurations are no longer obtainable. This is illustrated in figure 5 which shows contour plots of the energy distribution of the $B=3$ Skyrmion for different values of $s$ and fixed $\kappa$. For $s=0.5$ (figure 5a), the solution is rotationally symmetric and for $s=0.75$ and $s=1$ (figures $5 \mathrm{~b}$ and $5 \mathrm{c}$ respectively) the rotational symmetry of the solution is broken and only $\mathbb{Z}(2)$ symmetry remains. At $s=3$, no stable solution exists. The latter case is shown in figure $5 \mathrm{~d}$ which was taken while the distance between the individual Skyrmions kept growing.

The dependence of the Skyrmion solutions on the value of $\kappa$ with fixed $s$ has also been studied. Qualitatively, for small $\kappa$ the minimal energy configurations are rotationally-symmetric. Increasing its value results in an increasingly larger rotational symmetry breaking. This is illustrated in figure 6 . 


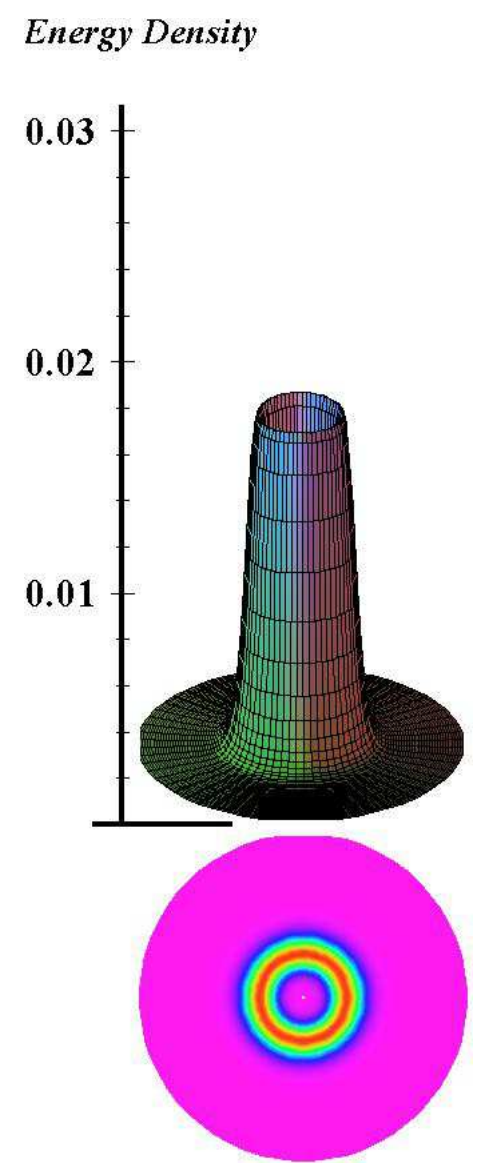

(a) $s=0.5$

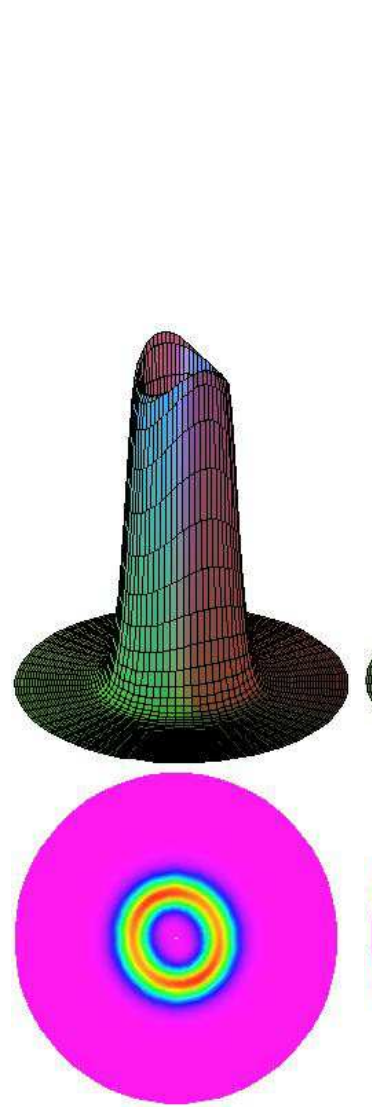

(b) $s=0.75$

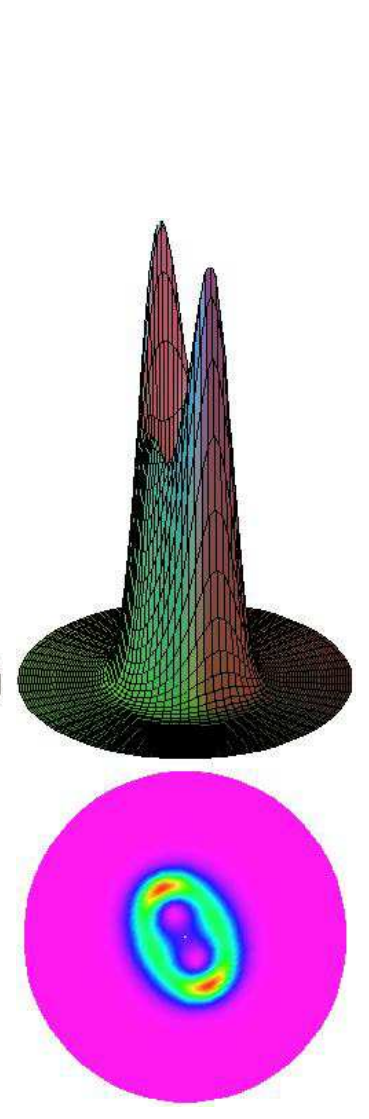

(c) $s=1$

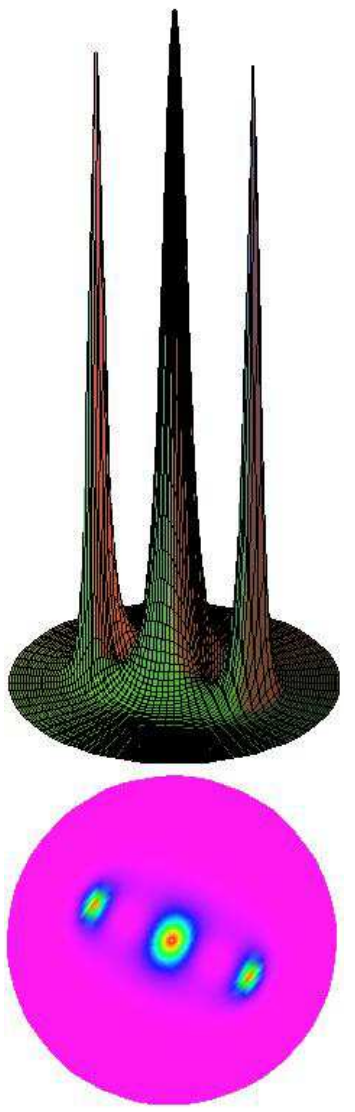

(d) $s=3$

Figure 5: Energy densities and corresponding contour plots (ranging from violet - low density to red - high density) of the $B=3$ Skyrmion for fixed $\kappa\left(\kappa^{2}=0.01\right)$ and varying $s$. The energy is plotted as function of the angle $\theta$ and of the scaled radial coordinate $\tilde{r}$ (eq. (44)). In the $s=0.5$ case, the minimal energy configuration is rotationally symmetric, corresponding the three one-Skyrmions on top of each other. For $s=0.75$ and $s=1$ the solutions exhibit only $\mathbb{Z}(2)$ symmetry, corresponding to partially-overlapping one-Skyrmions. For $s=3$ no stable solution exists and the individual Skyrmions are drifting apart.

To better appreciate the effects of the parameters of the model on the obtained solutions, we introduce the following measure for the breaking of rotational symmetry:

$$
\Delta^{2}=\int\left(\frac{1}{2 B} \int \mathcal{B}(r, \theta) r \mathrm{~d} r\right)^{2} \frac{d \theta}{2 \pi}-1,
$$

where $\mathcal{B}(r, \theta)$ is the charge density of the Skyrmion with rotationally-symmetric configurations corresponding to $\Delta^{2}=0$. In terms of this measure, the results presented above may be quantitatively characterized. In figure $7, \Delta^{2}$ is plotted as function of the potential parameter $s$ for various $\kappa$ values, illustrating the effects of these parameters on the breaking of rotational symmetry of the charge-three solutions. 


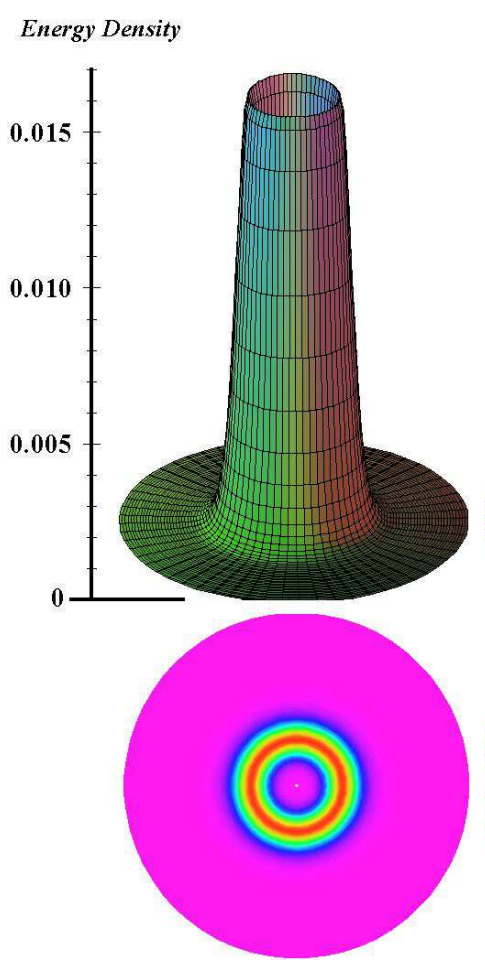

(a) $\kappa^{2}=0.01$



(b) $\kappa^{2}=0.1$
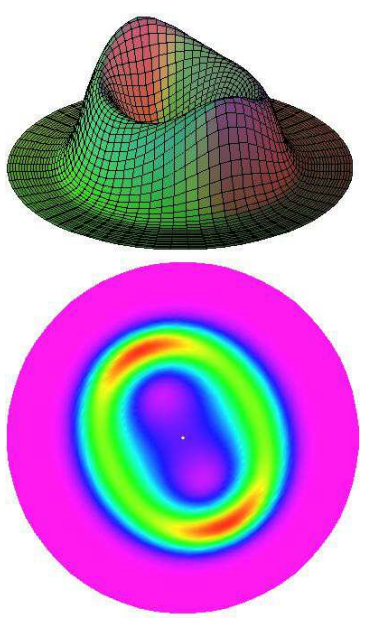

(c) $\kappa^{2}=0.25$
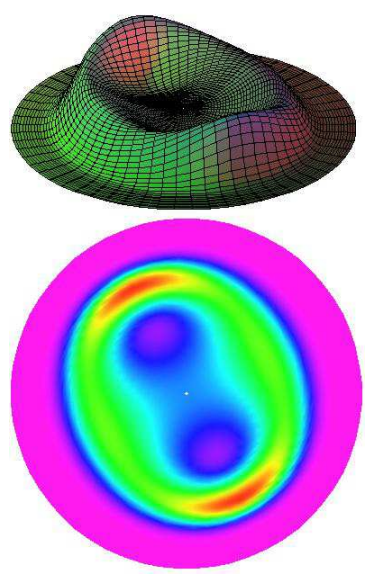

(d) $\kappa^{2}=1$

Figure 6: Energy densities and corresponding contour plots (ranging from violet - low density to red - high density) of the $B=3$ Skyrmion for fixed $s(s=0.5)$ and varying $\kappa$. At low $\kappa$, the minimal energy configuration is rotationally symmetric. As $\kappa$ is increased, breaking of rotational symmetry appears, and only $\mathbb{Z}(2)$ symmetry remains.

The $B=4$ and $B=5$ Skyrmion solutions behave similarly to the $B=3$ solutions, although (due to much larger computation time requirements) here the parameter space was not scanned as densely as with the lower charges. An illustrative example is given in figure 8, which shows the stable solutions that have been obtained in the $s=0.9$ case and the splitting of these Skyrmions into their constituents in the $s=4$ case. 


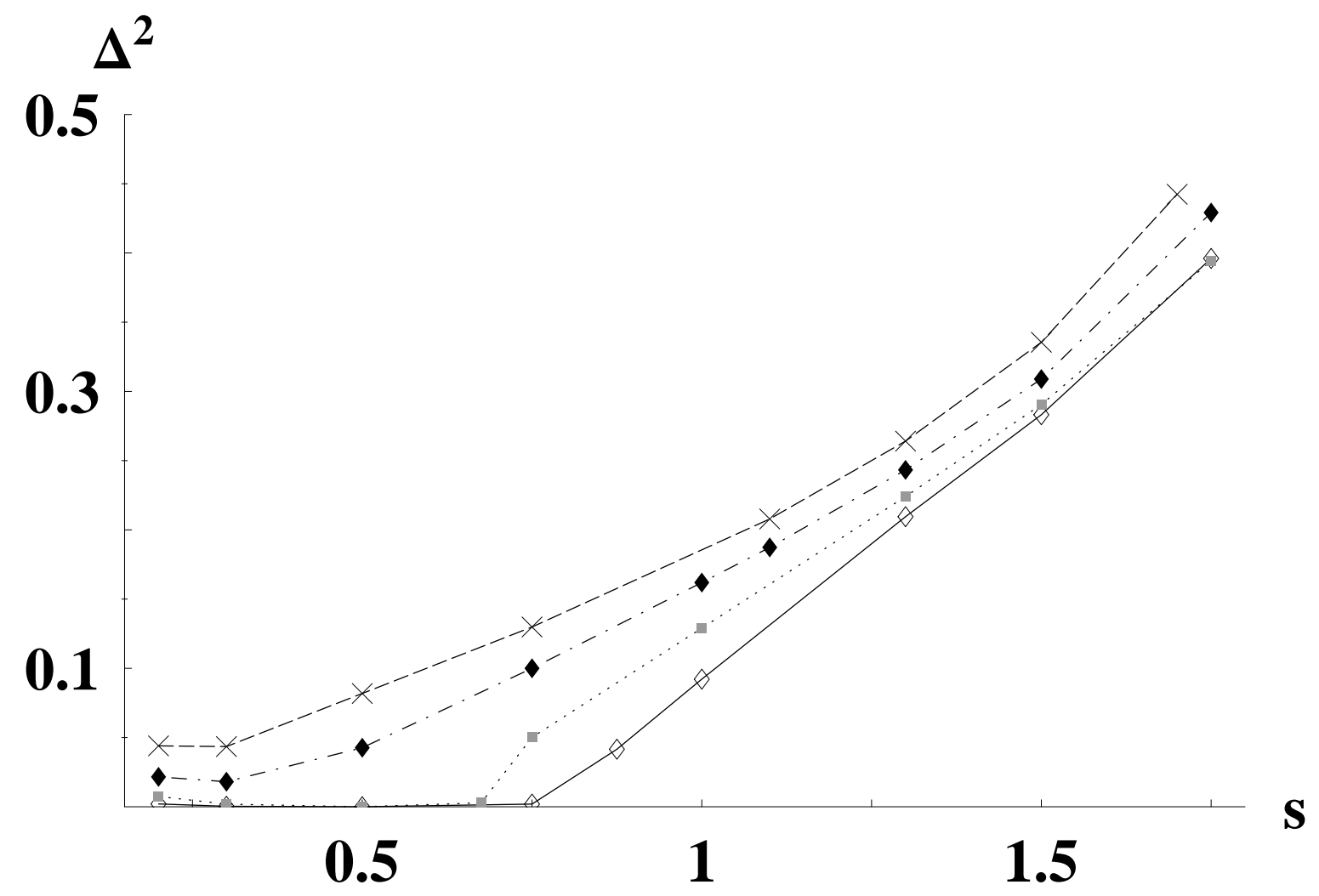

Figure 7: Symmetry-breaking measure $\Delta^{2}$, as a function of $s$ for various $\kappa$ values in the charge-three sector: $\kappa^{2}=0.01(\diamond), \kappa^{2}=0.05(\square), \kappa^{2}=0.25(\diamond)$, and $\kappa^{2}=1(\times)$. Breaking of rotational symmetry becomes more and more apparent as $s$ and $\kappa$ increase. The lines are to guide the eye. 


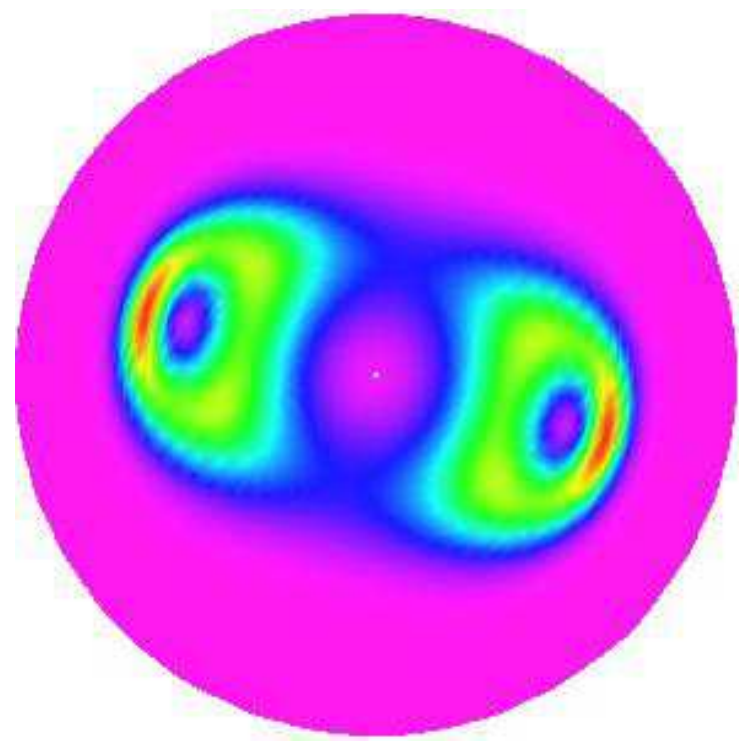

(a) $B=4, s=0.9$

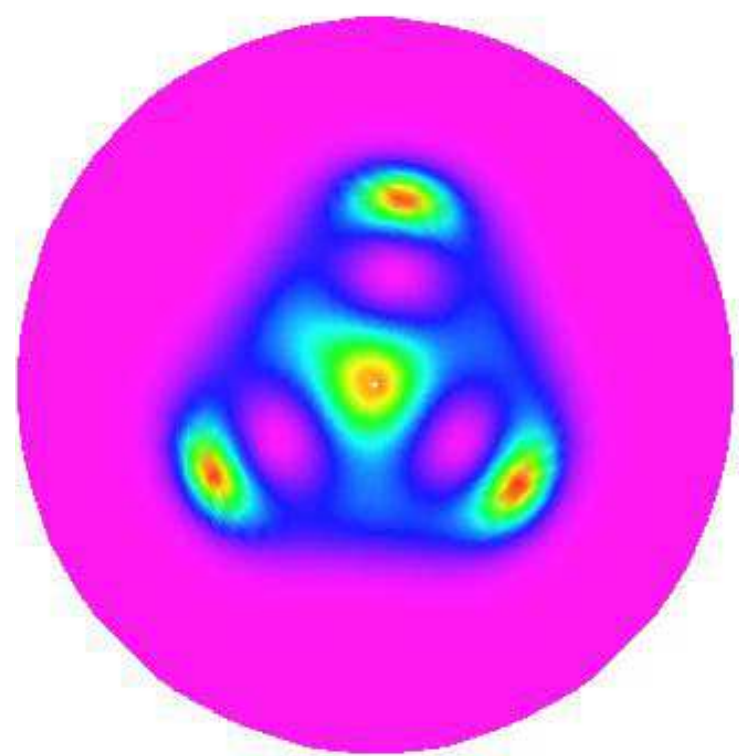

(c) $B=4, s=4$

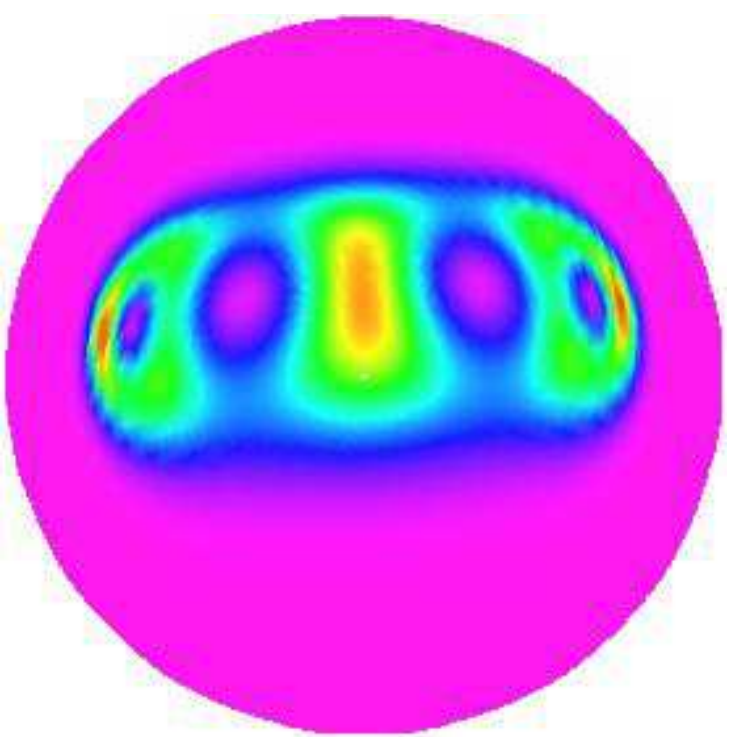

(b) $B=5, s=0.9$

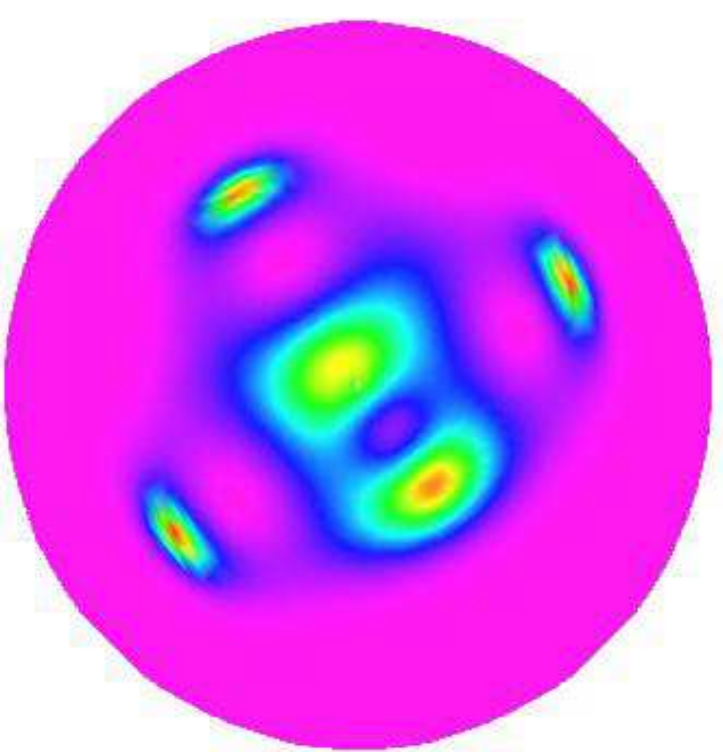

(d) $B=5, s=4$

Figure 8: Contour plots of the energy distributions (ranging from violet - low density to red - high density) of the $B=4$ and $B=5$ Skyrmions for $s=0.9$ and $s=4$ $\left(\kappa^{2}=0.1\right)$. In the lower $s$ region stable solutions exist; the upper figures show a $B=4$ Skyrmion in a bound state of two charge-two Skyrmions (left), and a $B=5$ Skyrmion in a two-one-two configuration. For values of $s$ higher than 2, the multi-Skyrmions split into individual one-Skyrmions constantly drifting apart (lower figures). 


\section{Summary and conclusion}

We have studied the static solutions of the baby Skyrme model for the one-parametric family of potentials $U=\mu^{2}\left(1-\phi_{3}\right)^{s}$. While Skyrmions with charge one were found to be stable and rotationally-symmetric for all tested values of $\kappa$ and $s$, the symmetries and stability of the minimal energy configurations of Skyrmions with higher charge were found to be strongly dependent on the parameter of the potential.

The charge-two Skyrmion was found to be ring-like and rotationally-symmetric but only for $s<2$, implying that in this regime, there exists a strong attraction between the individual Skyrmions. Above $s \approx 2$, the two-Skyrmion breaks apart into one-Skyrmions which repel each other and as a result drift infinitely apart.

For the $B>2$ Skyrmions, small values of $s$ yielded in general rotationally symmetric configurations; implying that the attraction between the individual Skyrmions is strong. Increasing the value of $s$ resulted in stable configurations with broken rotational symmetry. This type of solutions implies only a moderate attraction between the constituent Skyrmions, as this configuration may be viewed as three partiallyoverlapping charge-one Skyrmions in a row. As in the $B=2$ case, for $s>2$ no stable configurations exist. This is a consequence of a very strong repulsion between the Skyrmions which drives them infinitely apart from each other.

Altogether, it seems that $s$ may be viewed as a 'control' parameter for the strength of the attraction (small $s$ values) or repulsion (large $s$ values) between individual Skyrmions.

We also note here the locally stable solutions that have been obtained in the $B=2$ sector. We particularly note the locally stable two-Skyrmion solution that has been found in the 'holomorphic' $s=4$ case. This result stands in contradiction with previously known results [9] where it has been found that no stable solutions exist in this case. This discrepancy is not fully understood and might be due to the differences between the method used in [9] which is based on the time evolution of a system of two well separated Skyrmions sent towards one another, and the current one, in which multisolitons are obtained in a rather different approach, namely, by minimizing the energy functional of the system. Bearing in mind that the initial configuration in our minimization scheme is rotationally symmetric, it is possible that the minimization procedure ends up at a local rotationally-symmetric minimum, provided such a local minimum exists.

We verified that the results of our minimization procedure are robust with respect to changes in the grid size and in the initial configuration. They were also verified using the "simulated annealing". In most cases (with some notable exceptions as discussed above), our results were also found to agree with previously known results whenever a comparison was available. 


\section{Acknowledgments}

We thank Wojtek Zakrzewski for useful discussions. This work was supported in part

by a grant from the Israel Science Foundation administered by the Israel Academy of Sciences and Humanities.

\section{References}

[1] Skyrme T H R 1961 Proc. Roy. Soc. A260 127

[2] Skyrme T H R 1962 Nucl. Phys. 31556

[3] Adkins G S, Nappi C R and Witten E 1983 Nucl. Phys. B 228552

[4] For a review, see: Manton N S and Sutcliffe P M 2004 Topological Solitons (Cambridge: Cambridge University Press) p 350

[5] Piette B M A G, Schoers B J and Zakrzewski W J 1995 Z. Phys. C 65165

[6] Belavin A A and Polyakov A M 1975 JETP Lett. 22245

[7] Leese R A, Peyrard M and Zakrzewski W J 1990 Nonlinearity 3773

[8] Piette B M A G and Zakrzewski W J 1995 Chaos, Solitons and Fractals 52495

[9] Sutcliffe P M 1991 Nonlinearity 41109

[10] Piette B M A G, Schoers B J and Zakrzewski W J 1995 Nucl. Phys. B 439205

[11] Weidig T 1999 Nonlinearity 121489

[12] Eslami P, Sarbishaei M and Zakrzewski W J 2000 Nonlinearity 13, 1867

[13] Kirkpatrick S, Gellat C D and Vecchi M P 1983 Science 220671

[14] Geman S and Geman D 1984 IEEE Trans. Pattern Anal. Mach. Intell. 26721

[15] Hale M, Schwindt O and Weidig T 2000 Phys. Rev. E 624333 